\title{
Anti-phase action between the angular accelerations of trunk and leg is reduced in the elderly
}

Tomohisa Kato ${ }^{1}$, Shin-ichiro Yamamoto ${ }^{1}$, Tasuku Miyoshi ${ }^{1,2}$, Kimitaka Nakazawa ${ }^{3}$, Kei Masani $^{4,5}$, Daichi Nozaki ${ }^{6}$

1 Faculty of Systems Engineering, Shibaura Institute of Technology, Saitama, Japan

2 Graduate School of Engineering, Iwate University, Iwate, Japan

3 Department of Rehabilitation for Movement Functions, Research Institute, National Rehabilitation Center for Persons with Disabilities, Tokorozawa-City, Japan

4 Rehabilitation Engineering Laboratory, Toronto Rehabilitation Institute, Toronto, Ontario, Canada, M4G 3V9

5 Institute of Biomaterials and Biomedical Engineering, University of Toronto, Toronto, Ontario, Canada, M5S 3G9

6 Graduate School of Education, The University of Tokyo, Tokyo, Japan

Acknowledgments:

This work was supported by the Japan Space Forum, the Japan Science and Technology Agency, KAKENHI (\#20670008) and NEXT program (\#LS034). We thank to Matija Milocevic for assisting to prepare the manuscript.

Corresponding Author:

Kei Masani, $\mathrm{PhD}$

Rehabilitation Engineering Laboratory

Toronto Rehabilitation Institute

Toronto, Ontario, Canada, M4G 3V9

\author{
Daichi Nozaki, PhD \\ Graduate School of Education, The \\ University of Tokyo \\ 7-3-1 Hongo, Bunkyo-ku, Tokyo, JAPAN \\ Phone: $\quad+81-3-5841-3983 \quad$ Fax: \\ $+813-5879-8106$ \\ E-mail: nozaki@p.u-tokyo.ac.jp
}

Number of figures and tables: 5 Figures

Number of words in the text: 2995/3000 words from Introduction to Discussion

Number of words in the abstract: 248/250 words

(C) 2014. This manuscript version is made available under the Elsevier user license http://www.elsevier.com/open-access/userlicense/1.0/ 


\section{Abstract}

Quiet standing posture in humans has often been modeled as a single inverted pendulum pivoting around the ankle joint. However, recent studies have suggested that anti-phase action between leg and trunk segments plays a significant role in stabilizing posture by reducing the acceleration of the center of mass (COM) of the body. The aim of this study is to test the hypothesis that anti-phase action is attenuated in the elderly compared to the young. The anterior-posterior movements of leg and trunk segments were measured using 4 laser displacement sensors from 22 healthy young subjects (age range, 20-35 years) and 38 healthy elderly subjects (age range, 57-80 years) standing quietly for $30 \mathrm{~s}$ twice. To focus on the segmental action between trunk and legs, we applied constraints (i.e., wooden splints) on each segment. We found that the velocity and acceleration of the COM (standard deviation of the time series was evaluated) were significantly higher for the elderly subjects than for young subjects. The increase in the acceleration of the COM resulted not only from an increase in the angular acceleration of the segments but also from the reduction of their anti-phase relationship, as demonstrated by an index that quantifies the degree of cancellation between both segments. We conclude that the degree of anti-phase action between trunk and leg segments during quiet standing is smaller for elderly subjects than for young subjects, and that this change of the anti-phase action due to ageing resulted in increased COM acceleration in the elderly subjects.

Keywords: Posturography; Quiet standing; Aging; Intersegmental coordination; Hip strategy 


\section{Introduction}

Human bipedal stance is inherently unstable due to its configuration as an inverted pendulum. This instability is compensated by meticulous cooperative functions of visual, vestibular, and somatosensory systems [1]. Hence, the impairment of these functions due to aging should significantly affect the postural control in the elderly [2,3]. One of the most well known symptoms experienced by the elderly is an increased body sway during quiet standing, compared to young person [4] [5] [6].

Quiet standing posture is often approximated as a single-link inverted pendulum [7]. However, recent studies have pointed out that hip joint plays a significant role in postural maintenance even during quiet standing [8] [9] [10] [11]. For example, Aramaki et al. [8] demonstrated that the hip-joint movement is larger than the ankle joint movement during quiet standing. Therefore, it is necessary to analyze the coordination of the two segments when we investigate the ageing of postural control during quiet standing. Indeed, Accornero et al. [12] demonstrated the change of coordination due to ageing, i.e., the link between trunk and leg segments is enhanced in the elderly compared to the young.

Aramaki et al. [8] also demonstrated that, although the hip-joint action is considerable, its angular acceleration is in an anti-phase relationship with the ankle-joint's angular acceleration, which contributes to reducing the center of mass (COM) acceleration. This mechanism is thought to work by the anti-phase action between trunk and leg segments canceling out each other's acceleration. Such 
tightly controlled segmental action is widely seen in various human movements and it is intensively studied. The segmental action in human movements is theorized as the Minimum Intervention Principle [13] and is similarly conceptualized in the UnControlled Manifold concept (UCM) [14]. The UCM has revealed that body segments are coordinated to stabilize the COM position during standing [14]. The uniqueness of the finding by Aramaki et al. [8] was that the anti-phase action between trunk and leg segments was seen in their angular accelerations, and that such coordinated segmental action was mathematically proven to reduce the COM acceleration. Recently, Hsu et al. [15] investigated the ageing effect on the recovery from an unexpected perturbation during standing using UCM, and found that the elderly reduce the coordination of trunk and leg segments. Considering that the COM acceleration is higher in the elderly [6], we can hypothesize that the anti-phase relationship between the accelerations of trunk and leg segments is attenuated in the elderly, and that the COM acceleration is larger in the elderly than the young due to this ageing in the segmental coordination.

Therefore, the purpose of this study was to test this hypothesis by investigating trunk and leg segmental motions during quiet unperturbed standing for the elderly and the young. We introduced an index to quantify the anti-phase action based on a comparison of variability of COM acceleration between empirical data and theoretical data eliminating covariance of segmental motions. To focus on the segmental action between trunk and leg, we applied constraints, i.e., wooden splints, on each segment. 


\section{Methods}

\subsection{Subjects}

Twenty-two healthy young (age range, 20-35 years; 13 male and 9 female) and 38 healthy elderly (age range, 57-80 years; 2 male and 36 female) subjects participated in the experiment with informed consent. They had no medical history or signs of neurological disorders. The experimental procedure was approved by the ethical committee of our research institute. As the result of recruitment, there was an unequal gender distribution in each age group.

\subsection{Experimental protocol}

The barefoot subjects were asked to stand quietly for $30 \mathrm{~s}$ with their eyes open either on a force plate (9281B, Kistler, Switzerland) or on a floor. Two trials were performed for each subject. Note that the force plate recording was added in the later phase of data collection for 16 young and 21 elderly subjects for the purpose of measuring the center of pressure (COP). In order to focus the analysis on the motion in the sagittal plane, the intermalleolar distance was set to $10 \mathrm{~cm}$, because narrow stance width $(<8 \mathrm{~cm})$ increases lateral sway [16]. We also used 3 wooden splints that were strapped to the back of the subjects at the forehead and pelvis for the upper body, and above and below each knee for the legs (Fig.1A) so that motion was restricted to only the hip and ankle joints. Four CCD laser sensors (LK-2500, Keyence, Japan) were used to measure the anterior-posterior 
displacement at 4 locations of the body $\left(l_{1}-l_{4}\right.$ in Fig.1A), and the anterior-posterior position of the $\mathrm{COP}\left(X_{\mathrm{COP}}\right)$ was simultaneously recorded using the force plate (only for the subjects who stood on the force platform).

\subsection{Data processing}

The signals were A/D converted with a sampling frequency of $100 \mathrm{~Hz}$ (WE7251, Yokogawa Electric, Japan) and then low-pass filtered with a cut off frequency $=5 \mathrm{~Hz}$ using a fourth-ordered Butterworth filter with zero phase lag [17]. Each of the two trials was analyzed separately, and the average values were used to represent the subject's outcome.

The displacement measured by the laser sensors was then converted into angular displacements with an approximation as (Fig.1A): $\theta_{l} \approx\left\{\left(l_{2}-l_{2 \text { cal }}\right)-\left(l_{1}-l_{1 \text { cal }}\right)\right\} / h_{1}$ and $\theta_{t} \approx\left\{\left(l_{4}-l_{4 \text { cal }}\right)\right.$ - $\left.\left(l_{3}-l_{3 \text { cal }}\right)\right\} / h_{2}$, where $\theta_{l}$ and $\theta_{t}$ represent, respectively, the angles of trunk and leg segments, $l_{1-4}$ represent the distances from splints to each sensor surface, $l_{1-4 \mathrm{cal}}$ represent the distances from the calibration line and each sensor surface, and $h_{1}$ and $h_{2}$ represent the distance between the sensors \#1 and \#2 and between the sensors \#3 and \#4, respectively.

\subsection{Estimation of the position of the COM}

We modeled the body during quiet standing as 2 segments rotated around the ankle and hip joints

(Fig.1B). We measured body mass, length of leg, and length of trunk for each subject. Then, the mass 
and location of the center of mass for each segment were estimated using Winter [17]. Considering that the $\theta_{l}$ and $\theta_{t}$ during quiet standing were small, the anterior-posterior position of the $\operatorname{COM}\left(X_{\mathrm{COM}}\right)$ can be represented as:

$X_{\mathrm{COM}}=k_{1} \theta_{l}+k_{2} \theta_{t}$,

where $k_{1}=\left(m_{1} r_{1}+m_{2} L_{1}\right) /\left(m_{1}+m_{2}\right), k_{2}=m_{2} r_{2} /\left(m_{1}+m_{2}\right), m_{\mathrm{i}}$ is mass, $L_{\mathrm{i}}$ is length, and $r_{\mathrm{i}}$ is the distance from the center of mass of each segment to the adjacent joint of each segment ( $i=1$ and 2 for trunk and leg, respectively). Fig.1C demonstrates examples of $X_{\mathrm{COM}}$ and $X_{\mathrm{COP}}$. The slow component of $X_{\mathrm{COP}}$ coincides with the $X_{\mathrm{COM}}$ and the faster component oscillates around the $X_{\mathrm{COM}}$ [18]. The agreement of slow component between $X_{\mathrm{COM}}$ and $X_{\mathrm{COP}}$ indicates that the estimation of the $X_{\mathrm{COM}}$ using standard anthropometric data was sufficiently reliable.

The angular velocity $\left(\dot{\theta}_{l}\right.$ and $\left.\dot{\theta}_{t}\right)$, angular acceleration $\left(\ddot{\theta}_{l}\right.$ and $\left.\ddot{\theta}_{t}\right)$, velocity $\left(\dot{X}_{\mathrm{COM}}\right)$, and acceleration $\left(\ddot{X}_{\mathrm{COM}}\right)$ of the COM were calculated by differentiating the data. The standard deviation (SD) of each variable was calculated for each trial to evaluate the variability of fluctuation of each variable.

\subsection{Cancellation Index (CI)}

According to Aramaki et al. [8], the $\ddot{X}_{\mathrm{COM}}$ during quiet standing for young subjects is kept small by anti-phase action of angular acceleration between hip and ankle joints. Eq. (1) implies that the trajectory of $\ddot{\theta}_{l}$ and $\ddot{\theta}_{t}$ when the $\ddot{X}_{\mathrm{COM}}$ is zero can be represented as a line with negative slope 
$-k_{2} / k_{1}$ on the $\left(\ddot{\theta}_{l}, \ddot{\theta}_{t}\right)$ plane (Fig.2). As long as the angular accelerations changed on this line, the $\ddot{X}_{\mathrm{COM}}$ should be small even when the angular accelerations change considerably (Fig.2A). Naturally, the increase in the variability of both angular accelerations introduces the increase in the variability of $\ddot{X}_{\text {COM }}$ (Fig.2B). However, the same level of increase in variability of $\ddot{X}_{\mathrm{COM}}$ may arise if the angular accelerations of both segments lose their anti-phase relationship (Fig.2C). To evaluate these effects, we developed a mathematical index as follows. The SD of $\ddot{X}_{\mathrm{COM}}$ can be expressed as:

$$
\operatorname{SD}\left(\ddot{X}_{\mathrm{COM}}\right)=\sqrt{k_{1}^{2} \operatorname{var}\left(\ddot{\theta}_{l}\right)+k_{2}^{2} \operatorname{var}\left(\ddot{\theta}_{t}\right)+2 k_{1} k_{2} \operatorname{cov}\left(\ddot{\theta}_{l}, \ddot{\theta}_{t}\right)}
$$

where $\operatorname{var}(\mathrm{x})$ and $\operatorname{cov}(\mathrm{x}, \mathrm{y})$ represent, respectively, the variance of $\mathrm{x}$ and the covariance of $\mathrm{x}$ and $\mathrm{y}$. If the anti-phase relationship does not exist between $\ddot{\theta}_{l}$ and $\ddot{\theta}_{t}$, the $\operatorname{SD}\left(\ddot{X}_{\mathrm{COM}}\right)$ can be represented as $\sqrt{k_{1}^{2} \operatorname{var}\left(\ddot{\theta}_{l}\right)+k_{2}^{2} \operatorname{var}\left(\ddot{\theta}_{t}\right)}$ due to the absence of the covariance term of eq. (2). Therefore, the ratio (we will call this "Cancellation Index (CI)")

$$
\mathrm{CI}=\frac{\sqrt{k_{1}^{2} \operatorname{var}\left(\ddot{\theta}_{l}\right)+k_{2}^{2} \operatorname{var}\left(\ddot{\theta}_{t}\right)}}{\mathrm{SD}\left(\ddot{X}_{\mathrm{COM}}\right)}
$$

can evaluate the degree to which the anti-phase relationship contributes to reduce the $\operatorname{SD}\left(\ddot{X}_{\mathrm{COM}}\right)$.

The $\mathrm{CI}=1$ indicates that there is no cancellation, and the greater the CI, the greater the degree of cancellation. Note that, while the indexes used in the UnControled Manifold analysis $[14,15]$ can only reveal the tightness of the coupling between the corresponding joints, the analysis applied here can evaluate the contribution of the coupling effect between the two joints on the variability of the COM acceleration. 


\subsection{Statistical test}

Differences in variables between young and elderly subjects were tested by $t$-test. The significance level was set to $P<0.05$. The degree of freedom for each test was $d f=58$ in most of cases. Whenever we used a different degree of freedom, it has been noted. A log transformation was applied to $\mathrm{CI}$ before performing the statistical analysis.

\section{Results}

\subsection{Variability of postural sway}

No significant difference was observed between the young and elderly subjects for the SD of the angular motions $(P=0.453$ and $P=0.469$ for leg and trunk angular displacement; $P=0.090$ and $P$ $=0.392$ for leg and trunk angular velocity)(Fig.3A,B) except for the angular acceleration exhibiting significantly higher SD for the elderly subjects $(P=0.0004$ and $P=0.033$ for leg and trunk, respectively; Fig.3C). Although no significant difference was observed between the young and elderly subjects for the $\mathrm{SD}\left(X_{\mathrm{COP}}\right)(P=0.466)$ and $\mathrm{SD}\left(X_{\mathrm{COM}}\right)(P=0.170)\left(\right.$ Fig.3D), both $\mathrm{SD}\left(\dot{X}_{\mathrm{COM}}\right)(P=$ $0.028)$ and $\operatorname{SD}\left(\ddot{X}_{\mathrm{COM}}\right)(P<0.0001)$ were significantly higher for the elderly subjects (Fig.3E,F).

\subsection{Anti-phase movement between trunk and leg segments}

Figure 4 demonstrates the relationship of the kinematics between leg and trunk segments. As reported in the previous study [8], there was no clear anti-phase relationship between trunk and leg 
angular displacements (Fig.4) both for young and elderly subjects. The anti-phase relationship was most clearly seen in the trajectory on the $\left(\ddot{\theta}_{l}, \ddot{\theta}_{t}\right)$ plane for the young subjects (Fig.4A), but such clear anti-phase relationships were not observed for the elderly subjects (in particular for Elderly \#2;

Fig.4B,C). The loss of the anti-phase relationship with aging was quantitatively reflected in the CI. CI was significantly higher than 1 for the young $(\mathrm{P}<0.0001, d f=21)$ and the elderly $(\mathrm{P}<0.0001, d f=$ 37) subjects. The CI was significantly lower $(P<0.0001$, Fig.5A) for the elderly subjects $(0.100 \pm$ $0.060)$ than for the young subjects $(0.227 \pm 0.091)$, indicating the cancellation effect due to anti-phase action was reduced in the elderly.

\subsection{Validity of the $C I$}

A technical problem when calculating the $\mathrm{CI}$ is how the coefficients $k_{1}$ and $k_{2}$ in eq. (1) were estimated. We estimated them using subject's anthropometric data [17], but a possible difference in the shape of segments between young and elderly subjects, which was not considered in this method, should influence the CI. Thus, we tried an alternative method; the $X_{\mathrm{COM}}$ obtained by low-pass filtering $X_{\mathrm{COP}}\left(\right.$ cutoff frequency $=0.8 \mathrm{~Hz}$ ) [19] was regressed by the least square method as $X_{\mathrm{COM}}=k_{1} \theta_{l}+k_{2} \theta_{t}$.

Then the regression coefficients $k_{1}$ and $k_{2}$ were used to calculate the CI. Similarly to the CI obtained by the original method (Fig.5A,C), the CI estimated by the alternative method exhibited a significantly lower value $(P=0.0003, d f=35)$ for the elderly $(0.113 \pm 0.112)$ than for the young subjects $(0.398 \pm$ 0.225) (Fig.5B). Therefore, our conclusion that the CI was significantly lower for elderly subjects was 
validated.

\section{Discussion}

\subsection{Single inverted pendulum model is not suitable}

Although the quiet standing posture tends to be approximated as a single-link inverted pendulum [7], recent studies have pointed out that the hip joint plays a significant role for postural maintenance even during quiet standing [8] [9] [10] [11]. If the single inverted pendulum model is valid for quiet standing, then the movement of the trunk segment should have been identical to that of the leg segment. However, we observed that the variability of the trunk segment rotation was larger than that of the leg segment, especially for angular acceleration (Fig. 3), indicating that the single inverted pendulum model is not necessarily valid and that two-linked approximation is more appropriate.

\subsection{Anti-phase action between leg and trunk segments}

The trunk segment rotation can be compensated by the opposite rotation of the leg segment. Therefore, the anti-phase relationship between trunk and leg segments is beneficial to reduce the resultant COM motion. In a previous study [8], this anti-phase action was found for young subjects between angular 
accelerations of trunk and leg segments. In the present study, we reconfirmed this previous observation for the young and the elderly subjects (Fig.4A, Fig.5A). It should be noted that the anti-phase relationship was not an artifact, because the trunk and leg angular displacements were measured independently in our experimental setting. If the measurements were dependent on each other, false anti-phase relationships would have been observed (see [8]).

\subsection{Reduced anti-phase action in the elderly}

It is worth noting that postural sway prominently increased with aging in the acceleration rather than angular displacement or velocity (Fig.3D-F). This was in accordance with the study by Masani et al. [6], who demonstrated that the difference in postural sway between young and elderly subjects was reflected more clearly in the acceleration of COM than in the COM displacement. As mentioned in the

Method section (Fig.2), the increase in $\operatorname{SD}\left(\ddot{X}_{\mathrm{COM}}\right)$ is due to 2 different factors: increases in angular accelerations of both segments (Fig.2B) and/or changes in their relationship (Fig.2C). Our results that the CI (Fig.5A) as well as the angular accelerations of both segments (Fig.3C) were significantly higher for elderly subjects indicates that the age-dependent increment of COM acceleration partly resulted from the breakdown of their anti-phase relationship.

\subsection{How does the ageing of anti-phase action arise?}

The anti-phase action was found only between the angular accelerations of the two segments 
and not between the angular displacements, which agrees with our previous study [8]. This indicates that only fast component of the postural sway co-varied between trunk and leg segments. Although the mechanism of the anti-phase action remains unclear at this stage, neural and mechanical factors could be considered to be the source of the anti-phase action. Therefore, the ageing of the anti-phase action found in the current study also remains unsolved but we could speculate the mechanism as follows.

Zhang et al. [10] reported that trunk and leg segmental movements were anti-correlated, especially in the frequency range higher than $1 \mathrm{~Hz}$, and argued that such anti-phase action was likely to be due to mechanical factors such as simple action-reaction forces between the two segments via joint structures, because blocking of various sensory information (e.g., vision or proprioception) did not alter the anti-phase action. A similar argument may be applicable for the difference in CI that was found between the young and elderly subjects in the present study. This is especially relevant considering that the anti-phase relationship is observed at around $2 \mathrm{~Hz}$ in the young subjects in our previous study [8].

Even though the abovementioned evidence might suggest that the mechanism of anti-phase action is due to mechanical factors, the finding in the present study that ageing affects the anti-phase action inspires us to link the anti-phase action with a neural mechanism. Postural control requires the central nervous system (CNS) to integrate all available sensory input to construct information about the position of the COM. It is likely that any impairment of sensory functions due to aging could significantly disadvantage such a complex control system in the elderly [2,3]. For example, the 
sensitivity of the foot sole of the elderly subjects is impaired compared to young subjects [21], which could lead to instability of balance in the elderly as the shear force acting on the foot sole would play a significant role in the maintenance of standing posture [22] [23] and the CNS can use sensory information from the foot sole to estimate the COM acceleration [24]. Further, a tendency of the elderly to use more muscle cocontractions among muscles in the elderly [25] could cause a reduction of the anti-phase action. Therefore, the ageing of the anti-phase relationship of angular accelerations reducing the COM acceleration might result from the impairment of sensory functions and/or neural strategy of using muscles during standing.

As the current study did not provide sufficient evidence directly proving the abovementioned arguments, we cannot conclude the cause of our finding in the current study. Further studies are definitely needed to examine the causes of the ageing of the anti-phase relationship between the angular acceleration of trunk and leg segments during quiet standing.

\subsection{Study Limitation}

We used splints to investigate the coordination between trunk and the leg segments. Although the subjects did not feel any difficulty in stabilizing the posture with splints, it should be recognized that there are several drawbacks to using splints. Due to the splints, it would be possible that the characteristics of the natural quiet standing were partly lost in our experimental procedure. Although further research will be needed to investigate to what extent our results hold true for normal 
standing without the splints, we believe that the analysis of two-segmented body movement can give us deeper insights into the postural control of a multi-linked body than previous studies in which human quiet standing is modeled as a one-segmented body.

\section{Conclusion}

We have demonstrated that the degree of anti-phase action between trunk and leg segments during quiet standing is smaller for elderly subjects than for young subjects, as quantified by the CI.

The current results imply that the ageing of the anti-phase action resulted in increased COM acceleration in the elderly subjects. We proposed that the CI is a useful method in evaluating the ageing of the postural coordination. 


\section{References}

[1] Dietz V. Human neuronal control of automatic functional movements: interaction between central programs and afferent input. Physiological Reviews. 1992;72:33-69.

[2] Shaffer SW, Harrison AL. Aging of the somatosensory system: a translational perspective. Physical Therapy. 2007;87(2):193-207.

[3] Sturnieks DL, St George R, Lord SR. Balance disorders in the elderly. Clinical Neurophysiology. 2008;38(6):467-478.

[4] Sheldon JH. The effect of age on the control of sway. Gerontologia Clinica. 1963;5:129-138.

[5] Prieto TE, Myklebust JB, Hoffmann RG, Lovett EG, Myklebust BM. Measures of postural steadiness: differences between healthy young and elderly adults. IEEE Transactions Biomedical Engineering. 1996;43(9):956-966.

[6] Masani K, Vette AH, Kouzaki M, Kanehisa H, Fukunaga T, Popovic MR. Larger center of pressure minus center of gravity in the elderly induces larger body acceleration during quiet standing. Neuroscience Letters. 2007;422(3):202-206.

[7] Gage WH, Winter DA, Frank JS, Adkin AL. Kinematic and kinetic validity of the inverted pendulum model in quiet standing. Gait \& Posture. 2004;19(2):124-132.

[8] Aramaki Y, Nozaki D, Masani K, Sato T, Nakazawa K, Yano H. Reciprocal angular acceleration of the ankle and hip joints during quiet standing in humans. Experimental Brain Research. 2001;136(4):463-473.

[9] Creath R, Kiemel T, Horak F, Peterka R, Jeka J. A unified view of quiet and perturbed stance: simultaneous co-existing excitable modes. Neuroscience Letters. 2005;377(2):75-80.

[10] Zhang Y, Kiemel T, Jeka J. The influence of sensory information on two-component coordination during quiet stance. Gait and Posture. 2007;26(2):263-271.

[11] Sasagawa S, Ushiyama J, Kouzaki M, Kanehisa H. Effect of the hip motion on the body kinematics in the sagittal plane during human quiet standing. Neuroscience Letters. 2009;450(1):27-31.

[12] Accornero N, Capozza M, Rinalduzzi S, Manfredi GW. Clinical multisegmental posturography: age-related changes in stance control. Electroencephalography and Clinical Neurophysiology. 1997;105:213-219.

[13] Todorov E, Jordan MI. Optimal feedback control as a theory of motor coordination. Nature Neuroscience. 2002;5(11):1226-1235.

[14] Hsu W-L, Scholz JP, Schöner G, Jeka JJ, Kiemel T. Control and estimation of posture during quiet stance depends on multijoint coordination. Journal of Neurophysiology. 2007;97(4):3024-3035.

[15] Hsu W-L, Chou L-S, Woollacott M. Age-related changes in joint coordination during balance recovery. Age (Dordrecht, Netherlands). 2013;35(4):1299-1309.

[16] Day BL, Steiger MJ, Thompson PD, Marsden CD. Effect of vision and stance width on human body motion when standing: implications for afferent control of lateral sway. Journal 
of Physiology. 1993;469:479-499.

[17] Winter DA. Biomechanics and Motor Control of Human Movement. 3rd edition ed. New Jersey: John Wiley \& Sons; 2004.

[18] Winter DA, Patla AE, Prince F, Ishac M, Gielo-Perczak K. Stiffness control of balance in quiet standing. Journal of Neurophysiology. 1998;80:1211-1221.

[19] Caron O, Faure B, Breniere Y. Estimating the centre of gravity of the body on the basis of the centre of pressure in standing posture. Journal of Biomechanics. 1997;30(11-12):1169-1171.

[20] Wolpert DM, Miall R, Ch., Kawato M. Internal models in the cerebellum. Trends in Cognitive Sciences. 1998;2(9):338-347.

[21] Perry SD. Evaluation of age-related plantar-surface insensitivity and onset age of advanced insensitivity in older adults using vibratory and touch sensation tests. Neuroscience Letters. 2006;392(1-2):62-67.

[22] Kavounoudias A, Roll R, Roll J-P. Foot sole and ankle muscle inputs contribute jointly to human erect posture regulation. Journal of Physiology. 2001;532:869-878.

[23] Meyer PF, Oddsson LI, De Luca CJ. The role of plantar cutaneous sensation in unperturbed stance. Experimental Brain Research. 2004;156(4):505-512.

[24] Morasso PG, Schieppati M. Can muscle stiffness alone stabilize upright standing? Journal of Neurophysiology. 1999;83:1622-1626.

[25] Benjuya N, Melzer I, Kaplanski J. Aging-induced shifts from a reliance on sensory input to muscle cocontraction during balanced standing. Journals of Gerontology Series A, Biological sciences and medical sciences. 2004;59(2):166-171. 


\section{Figure legends}

\section{Figure 1}

Schematic diagram of the experimental setup and definition of angular displacement of leg $\left(\theta_{l}\right)$ and trunk $\left(\theta_{t}\right)$ segments. A. The CCD sensors were used to measure the distance between splint and sensor. Using a tape measure, the distance between the splint and the central line of the body was also measured. Adding the distance to the data from sensors yielded $l_{1}, l_{2}, l_{3}$ and $l_{4}$. B. Two link model was used to obtain the relationship between the anterior-posterior position of center of body mass $\left(X_{\mathrm{COM}}\right)$ and $\theta_{l}$ and $\theta_{t}$. The $L, r$, and $m$ represent, respectively, the length of segment, the length between the joint and the center of mass of segment, and mass. C. Representative examples of estimated $X_{\mathrm{COM}}$ and $X_{\mathrm{COP}}$ (anterior-posterior position of center of pressure).

\section{Figure 2}

Dependence of variability of COM acceleration $\left(\ddot{X}_{\mathrm{COM}}\right)$ on distribution of leg $\left(\ddot{\theta}_{l}\right)$ and trunk angular acceleration $\left(\ddot{\theta}_{t}\right)$. Gaussian curve on each axis indicates the marginal distribution of the angular acceleration and that on the oblique line $\left(\ddot{X}_{\mathrm{COM}}=0\right.$, or $\left.k_{1} \ddot{\theta}_{l}+k_{2} \ddot{\theta}_{t}=0\right)$ distribution of the $\ddot{X}_{\mathrm{COM}}$.

A. The variability of $\ddot{X}_{\mathrm{COM}}$ when data is distributed around the line. B. The increase in the variability of both segment angular acceleration, with their relationship unchanged, induces an increase in the variability of $\ddot{X}_{\mathrm{COM}}$. C. However, changes in their relationship can result in the same amount of increase. 


\section{Figure 3}

Results of variability of postural sway (standard deviation of time series). A. Angular displacement. B. Angular velocity. C. Angular acceleration. D. Anterior-posterior displacement of the center of body mass $(\mathrm{COM})$ and the center of pressure $(\mathrm{COP})$. Note that, as the COP was not measured for all subjects, the number of subjects for this graph were $\mathrm{N}=16$ and $\mathrm{N}=21$ for young and elderly subjects, respectively. E. Anterior-posterior velocity of COM. F. Anterior-posterior acceleration of COM. Data are represented as mean $\pm \mathrm{SD}$.

\section{Figure 4}

Representative relationships of the angular displacement (left), angular velocity (middle), and angular accelerations (right) between leg and trunk segments for a young subject (A) and elderly subjects (B, C). The oblique lines in the right panels indicates the $\ddot{X}_{\mathrm{COM}}=0$ (i.e., $k_{1} \ddot{\theta}_{l}+k_{2} \ddot{\theta}_{t}=0$ ).

\section{Figure 5}

A. Result of the Cancellation Index (CI). B. The Cancellation Index (CI) was calculated using the data

of center of pressure. C. For comparison, the CI calculated using anthropometric data is shown for the subjects whose COP was measured. Data are represented as mean $\pm \mathrm{SD}$. 


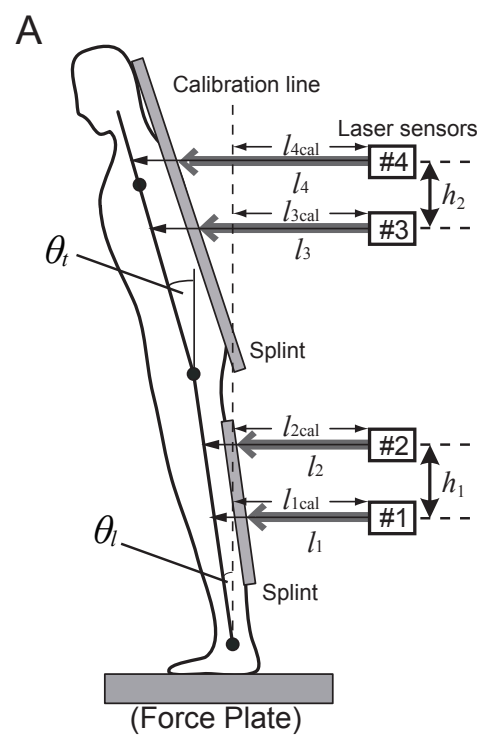

B

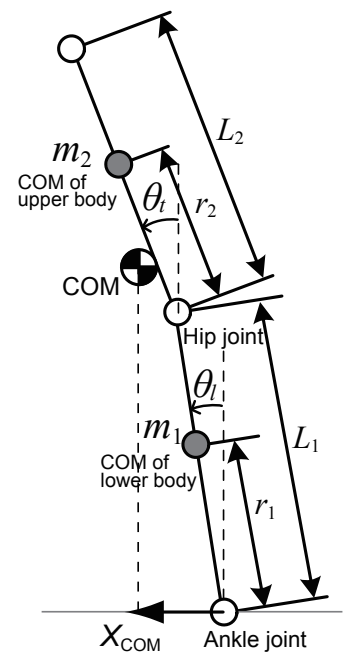

C

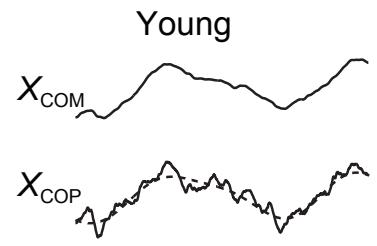

Elderly

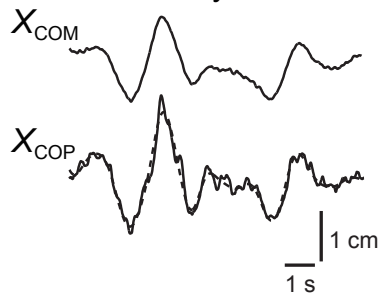

Figure 1: Kato et al. 

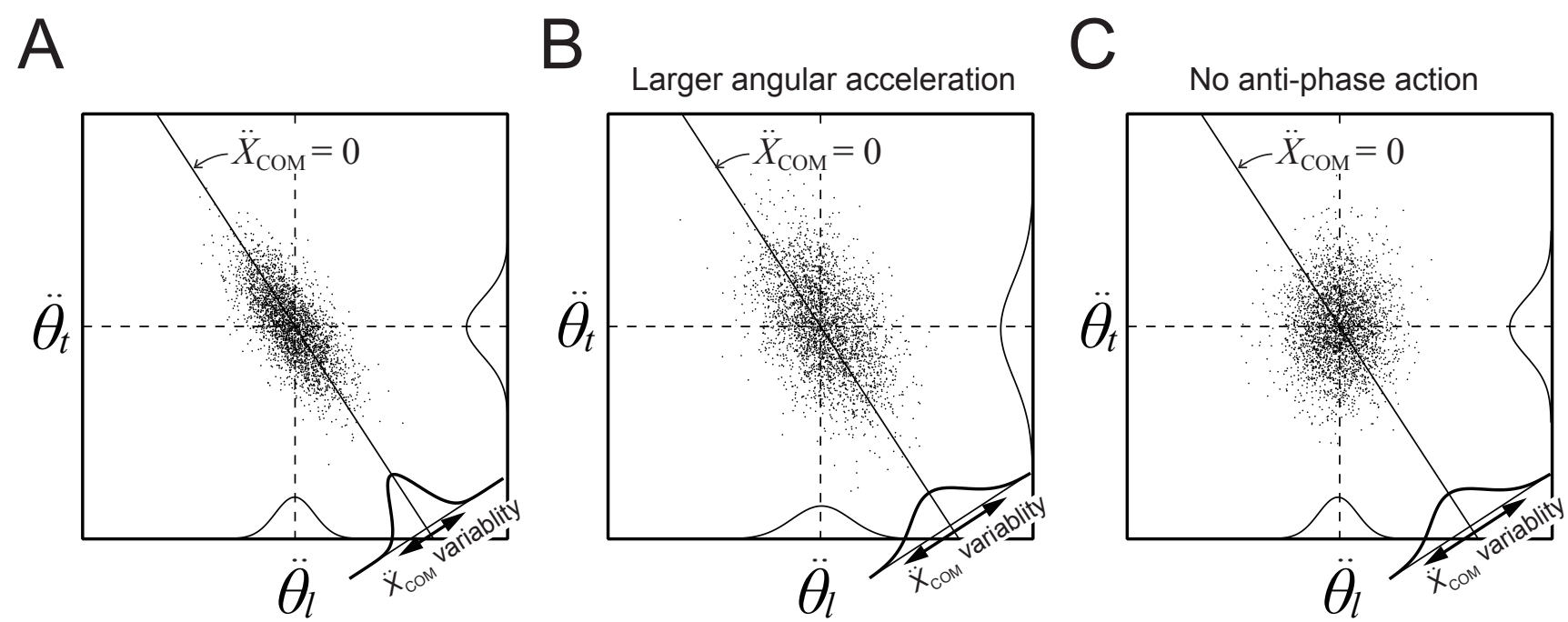

Figure 2: Kato et al. 

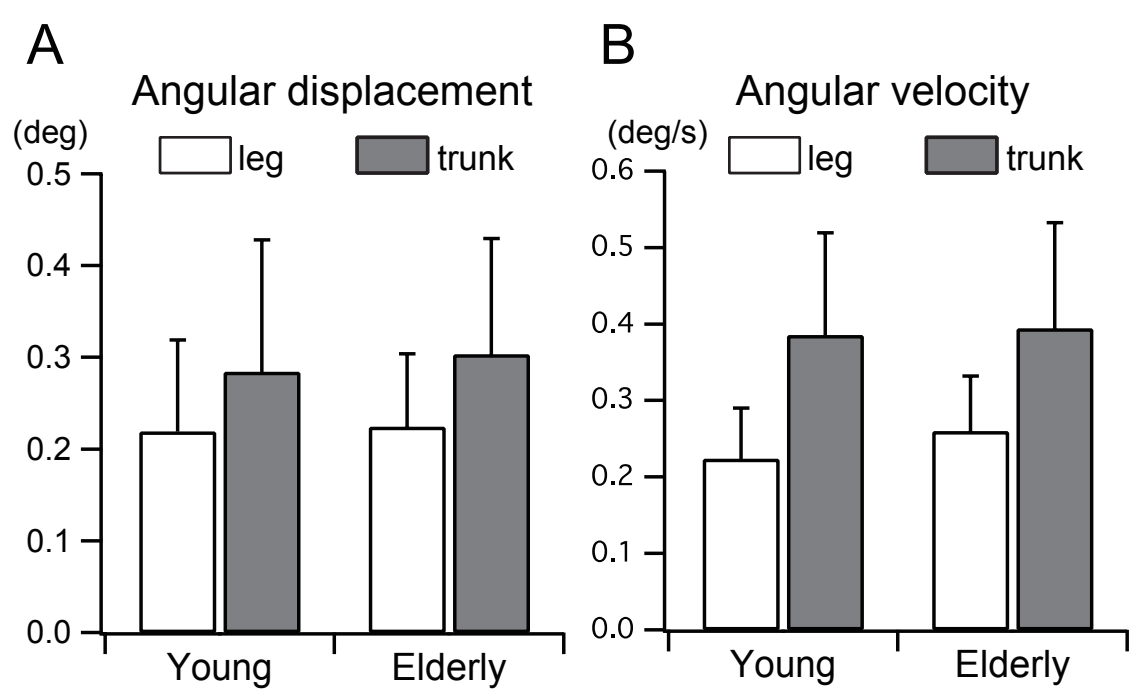

C Angular acceleration
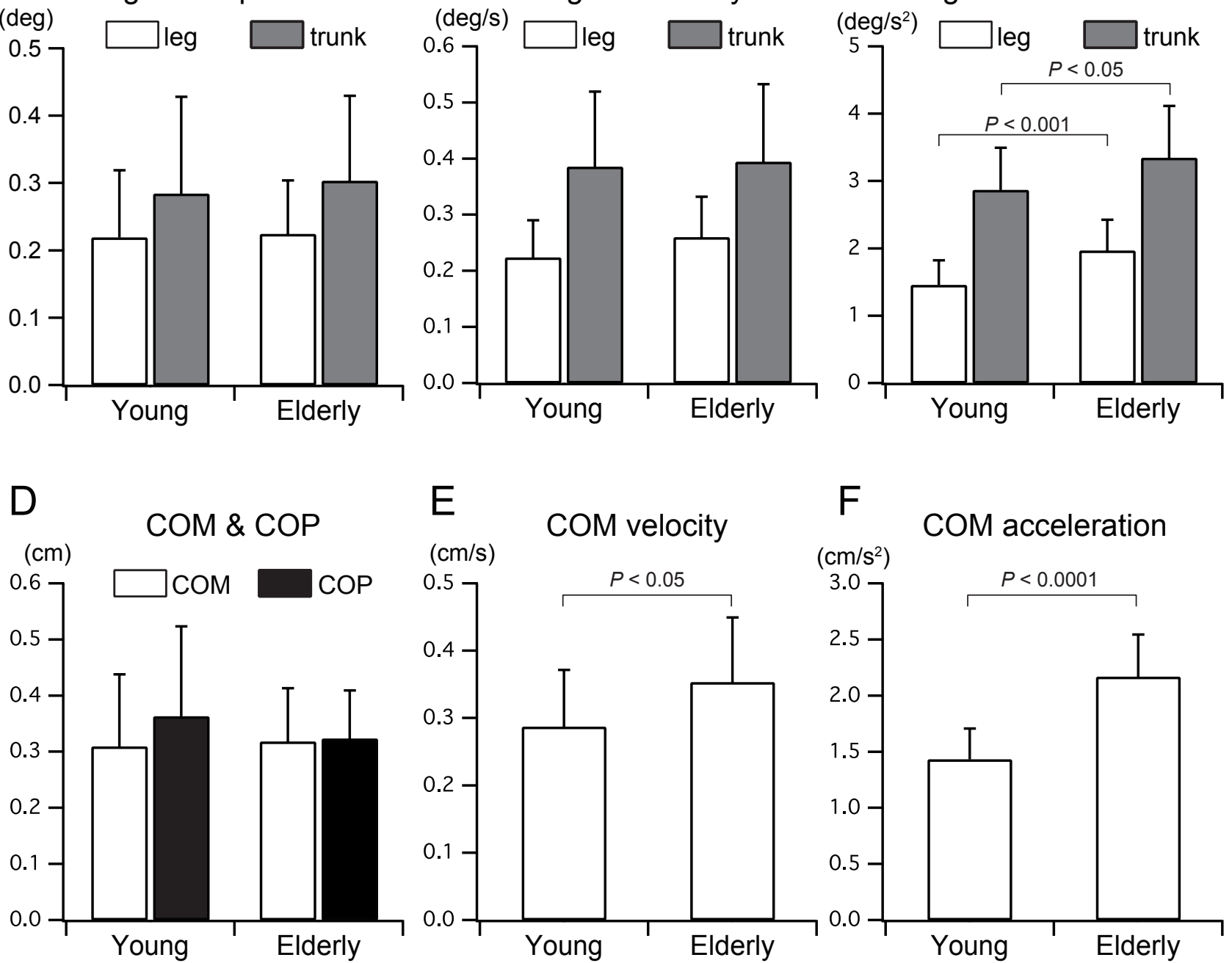

Figure 3: Kato et al. 
A. Young
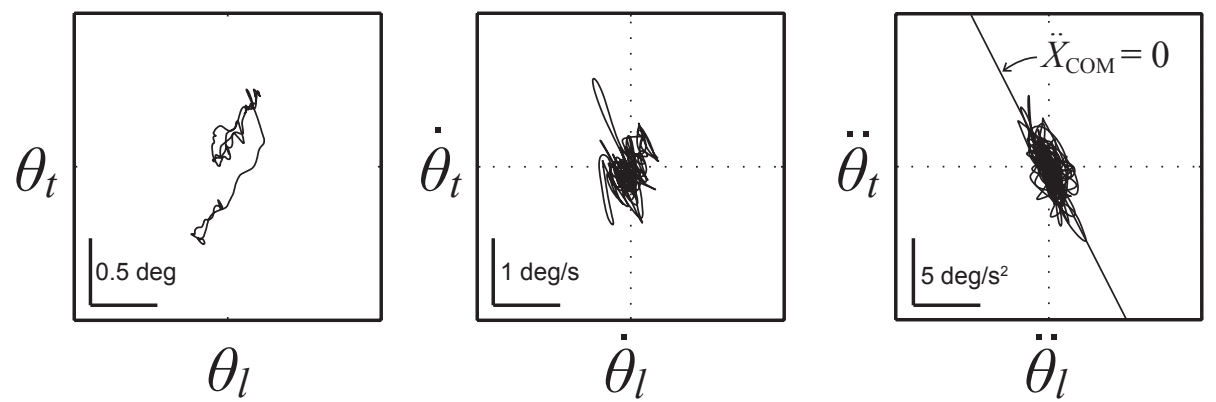

B. Elderly \#1
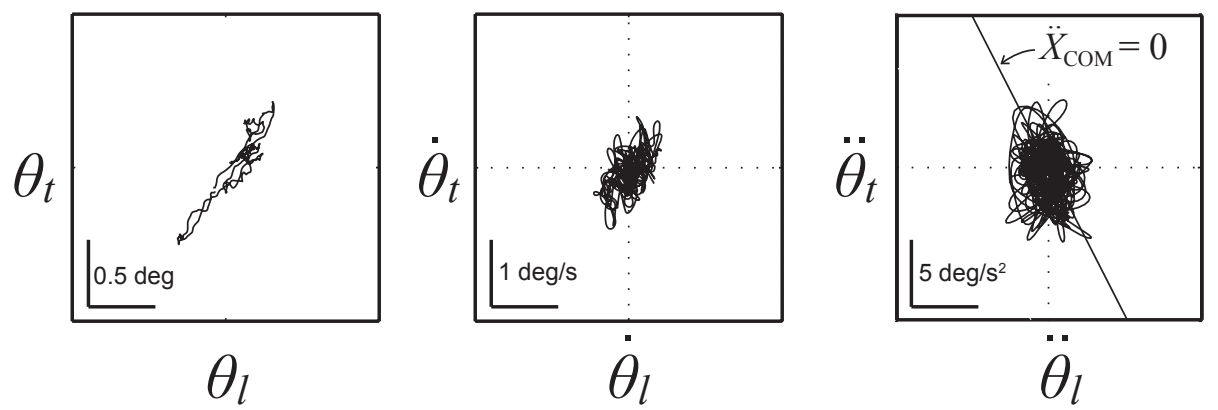

C. Elderly \#2
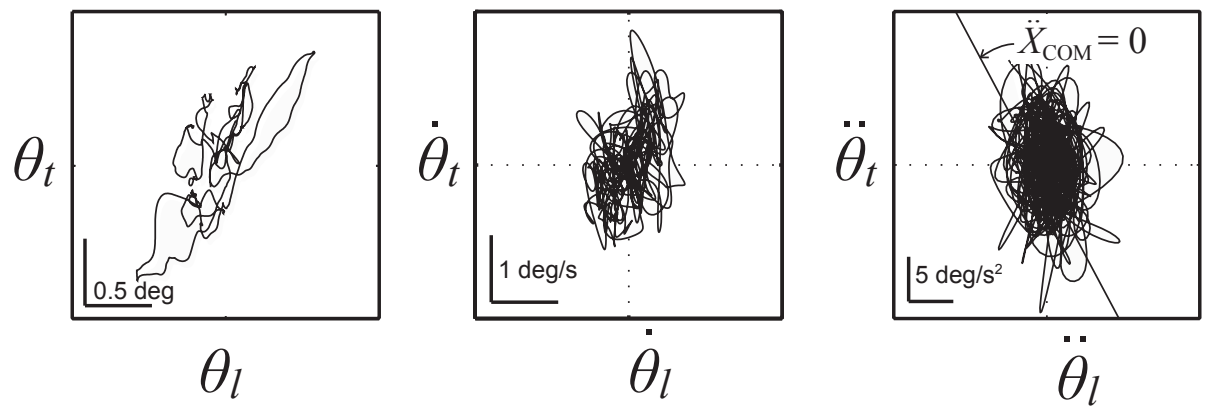

Figure 4: Kato et al. 


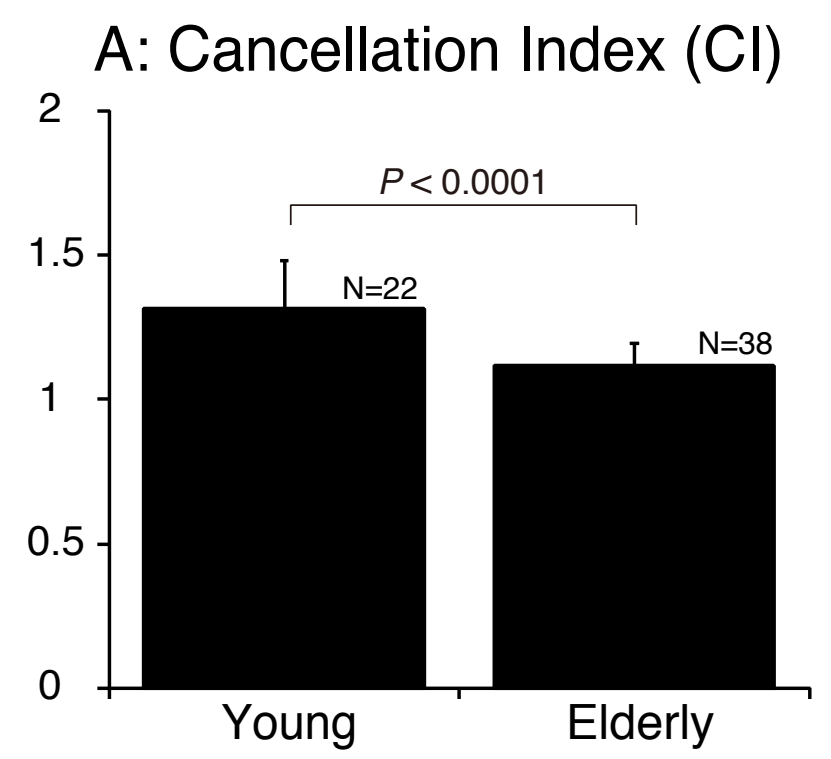

$\mathrm{B}$ : Cl obtained using COP

$\mathrm{C}: \mathrm{Cl}$
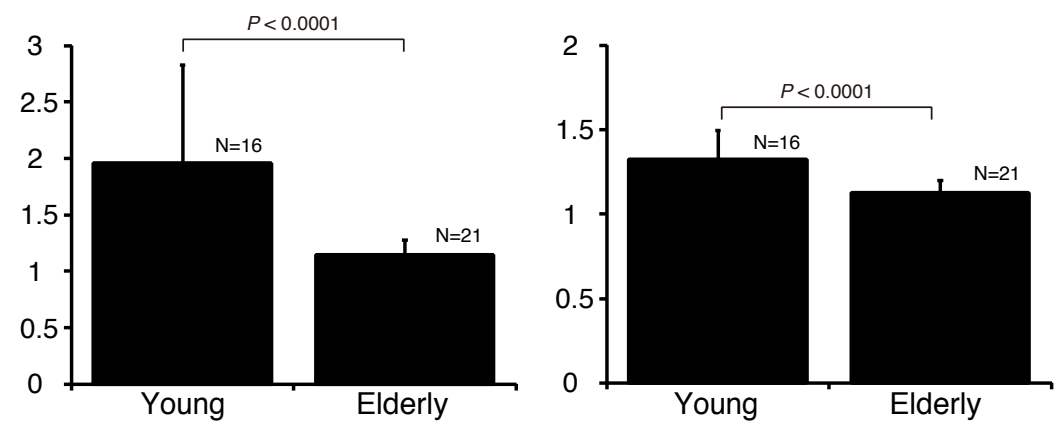

Figure 5: Kato et al. 